\title{
On a class of singular nonlinear first order partial differential equations
}

\author{
Hidetoshi TAHARA*
}

\begin{abstract}
In this paper, we consider a class of singular nonlinear first order partial differential equations $t(\partial u / \partial t)=F(t, x, u, \partial u / \partial x)$ with $(t, x) \in \mathbb{R} \times \mathbb{C}$ under the assumption that $F\left(t, x, z_{1}, z_{2}\right)$ is a function which is continuous in $t$ and holomorphic in the other variables. Under suitable conditions, we determine all the solutions of this equation in a neighborhood of the origin.
\end{abstract}

Key words and phrases: nonlinear partial differential equation, first order equation, Briot-Bouquet type.

2020 Mathematics Subject Classification Numbers: Primary 35F20; Secondary $35 \mathrm{~A} 01$.

\section{Introduction}

The ordinary differential equation

$$
t \frac{d u}{d t}=f(t, u) \quad \text { under } f(0,0)=0
$$

is called the Briot-Bouquet's ordinary differential equation. This was first studied by Briot-Bouquet [1]. In the analytic case, we have many references: Hille [5], Hukuhara-Kimura-Matuda [6], Gérard [2], Iwasaki-KimuraShimomura-Yoshida [7, etc. In the non-analytic case, we have some references: Wintner [15], Sansone-Conti [12].

In this paper, as a generalization of (1.1) to partial differential equations, we will consider a class of singular nonlinear first order partial differential equations of the form

$$
t \frac{\partial u}{\partial t}=F\left(t, x, u, \frac{\partial u}{\partial x}\right)
$$

\footnotetext{
*Sophia University, Tokyo 102-8554, Japan. E-mail: h-tahara@sophia.ac.jp
} 
and determine all the solutions (including solutions with singularity at $t=0$ ) of the equation.

Let $t \in \mathbb{R}, x \in \mathbb{C}, z_{1} \in \mathbb{C}$ and $z_{2} \in \mathbb{C}$ be the variables. For $r>0$ we write $D_{r}=\{y \in \mathbb{C} ;|y|<r\}$, where $y$ represents $x, z_{1}$ or $z_{2}$. Let $T_{0}>0$, $R_{0}>0, \rho_{0}>0$, and set $\Omega=\left\{\left(t, x, z_{1}, z_{2}\right) \in\left[0, T_{0}\right] \times D_{R_{0}} \times D_{\rho_{0}} \times D_{\rho_{0}}\right\}$.

Let $F\left(t, x, z_{1}, z_{2}\right)$ be a function on $\Omega$, and let us consider the equation (1.2) under the following assumptions:

$\left.\mathrm{A}_{1}\right) F\left(t, x, z_{1}, z_{2}\right)$ is a continuous function on $\Omega$ which is holomorphic in the variables $\left(x, z_{1}, z_{2}\right) \in D_{R_{0}} \times D_{\rho_{0}} \times D_{\rho_{0}}$ for any fixed $t$.

A $) F(0, x, 0,0)=0$ on $D_{R_{0}}$.

A $) \frac{\partial F}{\partial z_{2}}(0, x, 0,0)=0$ on $D_{R_{0}}$.

In the case where $F\left(t, x, z_{1}, z_{2}\right)$ is a holomorphic function in a neighborhood of $\mathbb{C}_{t} \times \mathbb{C}_{x} \times \mathbb{C}_{z_{1}} \times \mathbb{C}_{z_{2}}$, this equation is called a Briot-Bouquet type partial differential equation in $t$, and it was investigated in details by Gérard-Tahara [3, 4] and Yamazawa [16]. See also Li [8] and Yamazawa [17].

We set

$$
\lambda(t, x)=\frac{\partial F}{\partial z_{1}}(t, x, 0,0) .
$$

In the case where $F\left(t, x, z_{1}, z_{2}\right)$ is only continuous in $t$, the existence and the uniqueness of the solution were establised under the condition $\operatorname{Re} \lambda(0,0)<0$ by Lope-Roque-Tahara [9] and Tahara [13. This concludes that in the case $\operatorname{Re} \lambda(0,0)<0$ the set of all the solutions of $(1.2)$ consists of one solution.

But, in the case $\operatorname{Re} \lambda(0,0)>0$ we have no results up to now. Thus, in this paper we will consider the following problem:

Problem 1.1. Determine all the solutions of (1.2) under $\left.\left.A_{1}\right) \sim A_{3}\right)$ and

$$
\operatorname{Re} \lambda(0,0)>0 .
$$

It should be noted that the $t$-continuous version of Cauchy-Kowalevsky theorem was first proved by Nagumo [10], and it was re-proved by Treves [14, Nirenberg [11] in a different way. Since then, to extend a holomorphic version to a $t$-continuous version has been one of problems in the theory of partial differential equations.

\section{Main results}

By expanding $F\left(t, x, z_{1}, z_{2}\right)$ into Taylor series in $\left(z_{1}, z_{2}\right)$ we can express (1.2) in the form

$$
t \frac{\partial u}{\partial t}=a(t, x)+\lambda(t, x) u+b(t, x) \frac{\partial u}{\partial x}+R\left(t, x, u, \frac{\partial u}{\partial x}\right)
$$


where $R\left(t, x, z_{1}, z_{2}\right)$ is a function of the form

$$
R\left(t, x, z_{1}, z_{2}\right)=\sum_{j+\alpha \geq 2} a_{j, \alpha}(t, x) z_{1}^{j} z_{2}^{\alpha} .
$$

Let $\mu(t) \in C^{0}\left(\left(0, T_{0}\right]\right)$ be a weight function on $\left(0, T_{0}\right]$ : this means that $\mu(t)$ is an increasing, continuous and positive value function on $\left(0, T_{0}\right]$ satisfying

$$
\int_{0}^{T_{0}} \frac{\mu(\tau)}{\tau} d \tau<\infty
$$

By this condition, we have $\mu(t) \longrightarrow 0$ (as $t \longrightarrow+0$ ).

In addition to $\left.A_{1}\right) \sim A_{3}$ ), we suppose $0<T_{0}<1$ and the following conditions:

c1) $|a(t, x)| \leq A t^{\mu}$ on $\left(0, T_{0}\right] \times D_{R_{0}}$ for some $A>0$ and $\mu>0$.

c2) $|\lambda(t, x)-\lambda(0, x)| \leq \Lambda \mu(t)$ on $\left(0, T_{0}\right] \times D_{R_{0}}$ for some $\Lambda>0$.

c3) $|b(t, x)| \leq \frac{B \mu(t)}{|\log t|}$ on $\left(0, T_{0}\right] \times D_{R_{0}}$ for some $B>0$.

For simplicity, for $W=[0, T] \times D_{R}$ or $W=(0, T] \times D_{R}$ we denote by $X_{0}(W)$ the set of all functions $f(t, x) \in C^{0}(W)$ which are holomorphic in $x$ for any fixed $t$. Then, we have $a(t, x), \lambda(t, x), b(t, x) \in X_{0}\left(\left[0, T_{0}\right] \times D_{R_{0}}\right)$, and also $a_{j, \alpha}(t, x) \in X_{0}\left(\left[0, T_{0}\right] \times D_{R_{0}}\right)$.

Similarly, we denote by $X_{1}(W)$ the set of all functions $f(t, x) \in C^{1}(W \cap$ $\{0<t<T\})$ satisfying $f(t, x) \in X_{0}(W)$ and $(t \partial f / \partial t)(t, x) \in X_{0}(W)$.

Let us define classes of functions in which we consider our equation (2.1).

Definition 2.1. (1) For $d>0, T>0$ and $R>0$ we denote by $X_{1}^{d}([0, T] \times$ $\left.D_{R}\right)$ the set of all $u(t, x) \in X_{1}\left([0, T] \times D_{R}\right)$ satusfying

$$
\max \{|u(t, x)|,|(\partial u / \partial x)(t, x)|\} \leq C t^{d} \quad \text { on }[0, T] \times D_{R}
$$

for some $C>0$.

(2) We set

$$
X_{1}^{d}=\underset{(T, R) \rightarrow(0,0)}{\lim _{1}} X_{1}^{d}\left([0, T] \times D_{R}\right)
$$

(where $\underset{\mathrm{lim}}{\longrightarrow}$ denotes the inductive limit). In other words, $X_{1}^{d}$ is the set of all germe of functions $u(t, x)$ belonging to $X_{1}^{d}\left([0, T] \times D_{R}\right)$ for some $T>0$ and $R>0$. By the definition, if two functions $u_{i}(t, x) \in X_{1}^{d}\left(\left[0, T_{i}\right] \times D_{R_{i}}\right)$ $(i=1,2)$ satisfy $u_{1}(t, x)=u_{2}(t, x)$ on $[0, \epsilon] \times D_{\delta}$ for some $\epsilon>0$ and $\delta>0$, we think that $u_{1}=u_{2}$ in $X_{1}^{d}$.

(3) We set also

$$
X_{1}^{+}=\bigcup_{d>0} X_{1}^{d}, \quad \text { and } \quad X_{1}^{(d)}=\bigcap_{0<a<d} X_{1}^{a} \quad(\text { for } d>0) .
$$


We note that $t^{\mu} \in X_{1}^{\mu}, t^{\mu}|\log t| \notin X_{1}^{\mu}$, but $t^{\mu}|\log t| \in X_{1}^{(\mu)}$. Let us define another class of functions.

Definition 2.2. For $T>0$ and $R>0$ we denote by $\mathscr{X}_{1}\left([0, T] \times D_{R}\right)$ the set of all $u(t, x) \in X_{1}\left([0, T] \times D_{R}\right)$ satisfying

$$
\varlimsup_{\rho \rightarrow+0}\left[\lim _{\sigma \rightarrow+0}\left(\frac{1}{\rho^{2}} \sup _{(0, \sigma) \times D_{\rho}}|u(t, x)|\right)\right]=0 .
$$

We set

$$
\mathscr{X}_{1}=\underset{(T, R) \rightarrow(0,0)}{\lim _{1}} \mathscr{X}_{1}\left([0, T] \times D_{R}\right) .
$$

It is clear that $X_{1}^{+} \subset \mathscr{X}_{1}$ holds. If $u(t, x) \in X_{1}\left([0, T] \times D_{R}\right)$ satisfies

$$
\lim _{t \rightarrow+0}\left(\sup _{x \in D_{R}}|u(t, x)|\right)=0
$$

we have $u(t, x) \in \mathscr{X}_{1}$. If $u(t, x)=x^{m}$ for some $m \in\{3,4, \ldots\}$ we have $u(t, x) \in \mathscr{X}_{1}$. Therefore, the class $\mathscr{X}_{1}$ is much wider than $X_{1}^{+}$.

Now, let us state our main results of this paper. We denote by $\mathcal{O}_{0}$ the set of all germs of holomorphic functions $\psi(x)$ at $x=0$. For $X=X_{1}^{(\mu)}, X_{1}^{+}$ or $\mathscr{X}_{1}$, we denote by $\mathcal{S}((2.1), X)$ the set of all solutions of (2.1) belonging to the class $X$.

Theorem 2.3. Suppose the conditions $\left.\left.\mathrm{A}_{1}\right) \sim \mathrm{A}_{3}\right),(1.3)$ and $\left.\left.\mathrm{c} 1\right) \sim \mathrm{c} 3\right)$. Then, we have the following results.

(1) We have $\mathcal{S}\left(\underline{(2.1)}, X_{1}^{(\mu)}\right) \neq \emptyset$.

(2) Take any $u_{0}(t, x) \in \mathcal{S}\left((2.1), X_{1}^{(\mu)}\right)$ and fix it. Then, for any $\psi(x) \in$ $\mathcal{O}_{0}$ the equation (2.1) has a unique solution $u(t, x) \in X_{1}^{+}$which is expressed in the form

$$
u(t, x)=u_{0}(t, x)+w(t, x)
$$

for some $w(t, x) \in X_{1}^{+}$satisfying

$$
\lim _{t \rightarrow+0}\left(t^{-\lambda(0, x)} w(t, x)\right)=\psi(x) \quad \text { uniformly near } x=0 .
$$

From now, we write this solution as $U(\psi)(t, x)$.

(3) If $u_{0}(t, x)$ is fixed, we have

$$
\left.\mathcal{S}\left(\underline{(2.1)}, \mathscr{X}_{1}\right)=\mathcal{S}(\underline{2.11}), X_{1}^{+}\right)=\left\{U(\psi) ; \psi(x) \in \mathcal{O}_{0}\right\} .
$$

Remark 2.4. In [9] and [13], in the case $\operatorname{Re} \lambda(0,0)<0$ we have discussed (2.1) under the condition

$$
|a(t, x)| \leq A \mu(t) \quad \text { on }\left[0, T_{0}\right] \times D_{R_{0}} .
$$

But, in the case $\operatorname{Re} \lambda(0,0)>0$ it is difficult to solve (2.1) under (2.3). This is the reason why we suppose a stronger condition $|a(t, x)| \leq A t^{\mu}$ instead of (2.3). 
Example 2.5. In order to understand the situation in Theorem 2.3, let us consider the following equation:

$$
t \frac{\partial u}{\partial t}=x t^{\mu}+\lambda u+\left(\frac{\partial u}{\partial x}\right)^{2} .
$$

In this case we have $a(t, x)=x t^{\mu}$ with $\mu>0, \lambda(t, x)=\lambda$ with $\lambda>0$, and $b(t, x)=0$.

(1) By a calculation we see that (2.4) has a solution $u_{0}(t, x)$ of the form: in the case $\lambda \neq \mu, 2 \mu$

$$
u_{0}(t, x)=\frac{x t^{\mu}}{\mu-\lambda}+\frac{t^{2 \mu}}{(\mu-\lambda)^{2}(2 \mu-\lambda)}
$$

in the case $\lambda=2 \mu$

$$
u_{0}(t, x)=\frac{x t^{\mu}}{\mu-\lambda}+\frac{t^{2 \mu}(\log t)}{(\mu-\lambda)^{2}}
$$

in the case $\lambda=\mu$

$$
u_{0}(t, x)=x t^{\mu}(\log t)+\frac{t^{2 \mu}(\log t)^{2}}{\mu}-\frac{2 t^{2 \mu}(\log t)}{\mu^{2}}+\frac{2 t^{2 \mu}}{\mu^{3}} .
$$

Thus, in the case $\lambda \neq \mu$ we have a solution $u_{0}(t, x) \in X_{1}^{\mu} \subset X_{1}^{(\mu)}$, and in the case $\lambda=\mu$ we have a solution $u_{0}(t, x) \in X_{1}^{(\mu)}$ (but, $u_{0}(t, x) \notin X_{1}^{\mu}$ ).

(2) Next, let us look for a solution of the form $u(t, x)=u_{0}(t, x)+w(t, x)$. Our equation (2.4) is reduced to an equation with respect to $w$ :

$$
t \frac{\partial w}{\partial t}=\lambda w+f(t) \frac{\partial w}{\partial x}+\left(\frac{\partial w}{\partial x}\right)^{2}
$$

with

$$
f(t)= \begin{cases}2 t^{\mu} /(\mu-\lambda), & \text { if } \lambda \neq \mu, \\ 2 t^{\mu}(\log t), & \text { if } \lambda=\mu .\end{cases}
$$

Then, for any $\psi(x) \in \mathcal{O}_{0}$ the equation (2.5) has a solution $W(\psi)$ of the form

$$
W(\psi)=\sum_{k \geq 0} \psi_{k}(\eta(t)+x) t^{(k+1) \lambda} \quad \text { with } \psi_{0}(x)=\psi(x)
$$

where

$$
\eta(t)=\int_{0}^{t} \frac{f(\tau)}{\tau} d \tau= \begin{cases}2 t^{\mu} /(\mu(\mu-\lambda)), & \text { if } \lambda \neq \mu, \\ 2 t^{\mu}(\log t) / \mu-2 t^{\mu} / \mu^{2}, & \text { if } \lambda=\mu,\end{cases}
$$

and $\psi_{k}(x)(k \geq 1)$ are determined by the following recurrence formula:

$$
\psi_{k}(x)=\frac{1}{k \lambda} \sum_{i+j=k-1} \psi_{i}^{(1)}(x) \psi_{j}^{(1)}(x) .
$$

(3) By Theorem 2.3 we have

$$
\mathcal{S}\left((\underline{2.4}), \mathscr{X}_{1}\right)=\mathcal{S}\left((\underline{2.4}), X_{1}^{+}\right)=\left\{u_{0}+W(\psi) ; \psi(x) \in \mathcal{O}_{0}\right\} \text {. }
$$


Example 2.6. The following example will help the readers' understanding of the condition (2.2). The equation

$$
t \frac{\partial u}{\partial t}=u-\left(\frac{\partial u}{\partial x}\right)^{2}
$$

has a solution $u=x^{2} / 4$, and in this case we have

$$
\varlimsup_{\rho \rightarrow+0}\left[\lim _{\sigma \rightarrow+0}\left(\frac{1}{\rho^{2}} \sup _{(0, \sigma) \times D_{\rho}}|u(t, x)|\right)\right]=\frac{1}{4}
$$

and so $x^{2} / 4 \notin \mathscr{X}_{1}$. If we take a function class $\mathscr{F}$ which contains $x^{2} / 4$, then we have $\mathcal{S}((2.6), \mathscr{F}) \neq \mathcal{S}\left((\underline{2.6}), X_{1}^{+}\right)$.

Thus, (2.2) will be an optimal condition for $\mathcal{S}\left(\underline{(2.1)}, \mathscr{X}_{1}\right)=\mathcal{S}\left(\underline{(2.1)}, X_{1}^{+}\right)$ to be valid.

As is seen in Example 2.6, some equations have solutions which do not belong to $\mathscr{X}_{1}$. At present, the author has no idea to treat such solutions.

The rest part of this paper is organized as follows. In the next $\S 3$, we show $\mathcal{S}\left(\left(\underline{2.1)}, X_{1}^{(\mu)}\right) \neq \emptyset\right.$ in the case $\mu>\operatorname{Re} \lambda(0,0)$, as an application of results in [9] and [13. In $\S 4$, we show $\mathcal{S}\left(\underline{(2.1)}, X_{1}^{(\mu)}\right) \neq \emptyset$ in the case $\mu \leq \operatorname{Re} \lambda(0,0)$. In $\S 5$, we reduce our problem to the case $a(t, x) \equiv 0$, and in the last $\S 6$, we show (2) and (3) of Theorem 2.3, in the case $a(t, x) \equiv 0$.

\section{Solvability in the case $\mu>\operatorname{Re} \lambda(0,0)$}

In this section, we consider the equation (2.1) under the following conditions:

$$
\begin{aligned}
& |a(t, x)| \leq A t^{\mu} \quad \text { on }\left[0, T_{0}\right] \times D_{R_{0}}, \\
& |b(t, x)| \leq B \mu(t) \quad \text { on }\left[0, T_{0}\right] \times D_{R_{0}},
\end{aligned}
$$

where $A>0, \mu>0, B>0$ and $\mu(t)$ is a weight function on $\left(0, T_{0}\right]$.

Theorem 3.1. Suppose (3.1), (3.2) and $0<\operatorname{Re} \lambda(0,0)<\mu$. Then, the equation (2.1) has a unique solution $u(t, x) \in X_{1}^{(\mu)}$.

Since this is an easy consequence of results in [9, 13, let us recall some results in [9, 13]. For a weight function $\mu(t)$ we define a function $\varphi(t)$ by

$$
\varphi(t)=\int_{0}^{t} \frac{\mu(\tau)}{\tau} d \tau, \quad 0<t \leq T_{0},
$$

and for $T>0, R>0$ and $r>0$ we set

$$
W_{T, R, r}=\left\{(t, x) \in[0, T] \times D_{R} ; \varphi(t) / r+|x|<R\right\} .
$$


Theorem 3.2. Suppose the conditions (3.2), $\operatorname{Re} \lambda(0,0)<0$, and

$$
|a(t, x)| \leq A \mu(t) \quad \text { on }\left[0, T_{0}\right] \times D_{R_{0}}
$$

for some $A>0$. Then, we have the following results.

(1)(Theorem 1.1 (with $\alpha=1$ ) in [9]). There are $T>0, R>0, r>0$ and $M>0$ such that equation (2.1) has a unique solution $u_{0}(t, x) \in X_{1}\left(W_{T, R, r}\right)$ satisfying

$$
\left|u_{0}(t, x)\right| \leq M \mu(t) \text { and }\left|\frac{\partial u_{0}}{\partial x}(t, x)\right| \leq M \mu(t) \quad \text { on } W_{T, R, r} .
$$

(2)(Theorem 2.2 in [13]). Let $u(t, x) \in X_{1}\left((0, T] \times D_{R}\right)$ be a solution of (2.1) with $T>0$ and $R>0$. If $u(t, x)$ satisfies

$$
\varlimsup_{\rho \rightarrow+0}\left[\lim _{\sigma \rightarrow+0}\left(\frac{1}{\rho^{2}} \sup _{(0, \sigma) \times D_{\rho}}|u(t, x)|\right)\right]=0
$$

we have $u(t, x)=u_{0}(t, x)$ on $(0, \epsilon] \times D_{\delta}$ for some $\epsilon>0$ and $\delta>0$, where $u_{0}(t, x)$ is the solution of (2.1) obtained in (1).

Proof of Theorem 3.1. Suppose (3.1), (3.2) and $0<\operatorname{Re} \lambda(0,0)<\mu$. Take any $d>0$ satisfying $\operatorname{Re} \lambda(0,0)<d<\mu$, and set $u=t^{d} w$. Then our equation (2.1) is written as an equation with respect to $w$ :

$$
\begin{aligned}
t \frac{\partial w}{\partial t}=t^{-d} a(t, x)+( & (t, x)-d) w+b(t, x) \frac{\partial w}{\partial x} \\
& +\sum_{j+\alpha \geq 2} a_{j, \alpha}(t, x) t^{d(j+\alpha-1)} w^{j}\left(\frac{\partial w}{\partial x}\right)^{\alpha} .
\end{aligned}
$$

If we set $\mu_{0}(t)=\mu(t)+t^{\mu-d}$, this $\mu_{0}(t)$ is also a weight function on $\left(0, T_{0}\right]$ and we have

$$
\begin{aligned}
& \left|t^{-d} a(t, x)\right| \leq A \mu_{0}(t) \quad \text { on }\left[0, T_{0}\right] \times D_{R_{0}}, \\
& |b(t, x)| \leq B \mu_{0}(t) \quad \text { on }\left[0, T_{0}\right] \times D_{R_{0}} .
\end{aligned}
$$

Since $\operatorname{Re}(\lambda(0,0)-d)<0$ holds, by (1) of Theorem 3.2 we have a solution $w(t, x) \in X_{1}\left([0, T] \times D_{R}\right)$ of (3.4) satisfying

$$
|w(t, x)| \leq M \mu_{0}(t) \text { and }\left|\frac{\partial w}{\partial x}(t, x)\right| \leq M \mu_{0}(t) \quad \text { on }[0, T] \times D_{R}
$$

for some $T>0, R>0$ and $M>0$. Thus, by setting $u(t, x)=t^{d} w(t, x)$ we have a solution $u(t, x) \in X_{1}^{d}$ of (2.1) satisfying

$$
\lim _{t \rightarrow+0}\left(\sup _{x \in D_{R}}\left|t^{-d} u(t, x)\right|\right)=0
$$


for some $R>0$.

The uniqueness of such a solution is verified as follows. Let $u(t, x) \in X_{1}^{d}$ be a solution of (2.1) satisfying (3.5). Set $w(t, x)=t^{-d} u(t, x)$ : then $w(t, x)$ is a solution of (3.4). By the condition (3.5) we can apply (2) of Theorem 3.2 to this situation. Hence we have the uniqueness of $w(t, x)$ which yields the uniqueness of $u(t, x)$.

Since $d$ is taken arbitrarily so that $\operatorname{Re} \lambda(0,0)<d<\mu$, we have the result $u(t, x) \in X_{1}^{(\mu)}$.

\section{Solvability in the case $0<\mu \leq \operatorname{Re} \lambda(0,0)$}

In this section, we will show

Theorem 4.1. Suppose (3.1), (3.2) and $0<\mu \leq \operatorname{Re} \lambda(0,0)$. Then, the equation (2.1) has a solution $u(t, x) \in X_{1}^{\mu}$ in the case $\mu<\operatorname{Re} \lambda(0,0)$ (resp. $u(t, x) \in X_{1}^{(\mu)}$ in the case $\left.\mu=\operatorname{Re} \lambda(0,0)\right)$.

Set

$$
L=t \frac{\partial}{\partial t}-\lambda(t, x)-b(t, x) \frac{\partial}{\partial x} .
$$

In the next subsection 4.1 we solve the Cauchy problem

$$
L w=g(t, x),\left.\quad w\right|_{t=T}=\psi(x) .
$$

Then, by using the solvability of this Cauchy problem and Theorem 3.1 , in subsection 4.2 we give a proof of Theorem 4.1,

\subsection{On the Cauchy problem (4.1)}

Let $\varphi(t)$ be as in (3.3). Take $0<T<T_{0}$ and $0<R<R_{1}<R_{0}$ so that

$$
R+B \varphi(T)<R_{1}, \quad R_{1}+B \varphi(T)<R_{0} .
$$

For $\left(t_{0}, x_{0}\right) \in[0, T] \times D_{R_{1}}$, we consider the initial value problem

$$
\frac{d x}{d t}=-\frac{b(t, x)}{t},\left.\quad x\right|_{t=t_{0}}=x_{0} .
$$

Since (3.2) is supposed, it is easy to see that (4.2) has a unique solution $x(t)$ on $[0, T]$ : we write this solution as $\phi\left(t ; t_{0}, x_{0}\right)$. Set

$$
\mathcal{D}=\bigcup_{x_{0} \in D_{R_{1}}}\left\{\left(t, \phi\left(t ; T, x_{0}\right)\right) ; 0<t \leq T\right\}
$$

It is also easy to see that

$$
(0, T] \times D_{R} \subset \mathcal{D} \subset(0, T] \times D_{R_{0}}
$$

and that $\mathcal{D} \cap\left\{t=t_{0}\right\}$ is an open subset of $\mathbb{C}$ for any $t_{0} \in(0, T]$. 
Proposition 4.2. Under the above situation, for any $g(t, x) \in X_{0}(\mathcal{D})$ and any holomorphic function $\psi(x)$ on $D_{R_{1}}$ the Cauchy problem (4.1) has a unique solution $w(t, x) \in X_{1}(\mathcal{D})$, and it is given by

$$
\begin{aligned}
w(t, x)= & \psi(\phi(T ; t, x)) \exp \left[-\int_{t}^{T} \frac{\lambda(s, \phi(s ; t, x))}{s} d s\right] \\
& -\int_{t}^{T} \exp \left[-\int_{t}^{\tau} \frac{\lambda(s, \phi(s ; t, x))}{s} d s\right] \frac{g(\tau, \phi(\tau ; t, x))}{\tau} d \tau .
\end{aligned}
$$

Proof. Let $w(t, x) \in X_{1}(\mathcal{D})$ be a solution of (4.1). Take any $x_{0} \in D_{R_{1}}$ and set

$$
\begin{aligned}
& w^{*}(t)=w\left(t, \phi\left(t ; T, x_{0}\right)\right), \\
& \lambda^{*}(t)=\lambda\left(t, \phi\left(t ; T, x_{0}\right)\right), \\
& g^{*}(t)=g\left(t, \phi\left(t ; T, x_{0}\right)\right) .
\end{aligned}
$$

Then, our problem (4.1) is written in the form

$$
t \frac{d w^{*}}{d t}-\lambda^{*}(t) w^{*}=g^{*}(t), \quad w^{*}(T)=\psi\left(x_{0}\right) .
$$

By integrating this we have

$$
\begin{aligned}
w^{*}(t)=\psi\left(x_{0}\right) \exp [ & \left.-\int_{t}^{T} \frac{\lambda^{*}(s)}{s} d s\right] \\
& -\int_{t}^{T} \exp \left[-\int_{t}^{\tau} \frac{\lambda^{*}(s)}{s} d s\right] \frac{g^{*}(\tau)}{\tau} d \tau .
\end{aligned}
$$

Therefore, we have

$$
\begin{aligned}
w\left(t, \phi\left(t ; T, x_{0}\right)\right)= & \psi\left(x_{0}\right) \exp \left[-\int_{t}^{T} \frac{\lambda\left(s, \phi\left(s ; T, x_{0}\right)\right)}{s} d s\right] \\
& -\int_{t}^{T} \exp \left[-\int_{t}^{\tau} \frac{\lambda\left(s, \phi\left(s ; T, x_{0}\right)\right)}{s} d s\right] \frac{g\left(\tau, \phi\left(\tau ; T, x_{0}\right)\right)}{\tau} d \tau .
\end{aligned}
$$

Take any $(t, x) \in \mathcal{D}$; then we have a unique $x_{0} \in D_{R_{1}}$ such that $x=$ $\phi\left(t ; T, x_{0}\right)$. In this case, we have $x_{0}=\phi(T ; t, x)$ and $\phi\left(s ; T, x_{0}\right)=\phi(s ; t, x)$ for $0<s \leq T$. Therefore, by replacing $x_{0}$ and $\phi\left(s ; T, x_{0}\right)$ by $\phi(T ; t, x)$ and $\phi(s ; t, x)$, respectively, in the above formula we finally obtain

$$
\begin{aligned}
w(t, x)= & \psi(\phi(T ; t, x)) \exp \left[-\int_{t}^{T} \frac{\lambda(s, \phi(s ; t, x))}{s} d s\right] \\
& -\int_{t}^{T} \exp \left[-\int_{t}^{\tau} \frac{\lambda(s, \phi(s ; t, x))}{s} d s\right] \frac{g(\tau, \phi(\tau ; t, x))}{\tau} d \tau .
\end{aligned}
$$

This proves (4.3) and also the uniqueness of the solution of (4.1).

To show the existence of a solution, we have only to notice that the right side of (4.3) is well-defined as a function in $X_{1}(\mathcal{D})$. Thus, the existence of a solution is also shown. 
Corollary 4.3. Suppose that $0<a_{0} \leq \operatorname{Re} \lambda(t, x)$ holds on $\mathcal{D}$. If $\psi(x)$ and $(\partial \psi / \partial x)(x)$ are bounded on $D_{R_{1}}$ and if $g(t, x) \in X_{1}^{\mu}(\mathcal{D})$ for some $\mu$ satisfying $0<\mu<a_{0}$, then the unique solution $w(t, x)$ of (4.1) belongs to $X_{1}^{\mu}(\mathcal{D})$.

Proof. Suppose that $|\psi(x)| \leq M$ on $D_{R_{1}}$ for some $M>0$, and $|g(t, x)| \leq G t^{\mu}$ on $\mathcal{D}$ for some $G>0$. Since

$$
\left|\exp \left[-\int_{t}^{T} \frac{\lambda\left(s, \phi\left(s ; T, x_{0}\right)\right)}{s} d s\right]\right| \leq \exp \left[-\int_{t}^{T} \frac{a_{0}}{s} d s\right]=\left(\frac{t}{T}\right)^{a_{0}}
$$

holds, by (4.3) we have

$$
\begin{aligned}
|w(t, x)| & \leq M\left(\frac{t}{T}\right)^{a_{0}}+\int_{t}^{T}\left(\frac{t}{\tau}\right)^{a_{0}} \frac{G \tau^{\mu}}{\tau} d \tau \\
& =M\left(\frac{t}{T}\right)^{a_{0}}+G t^{a_{0}}\left(\frac{-1}{\left(a_{0}-\mu\right) T^{a_{0}-\mu}}+\frac{1}{\left(a_{0}-\mu\right) t^{a_{0}-\mu}}\right) \\
& \leq M\left(\frac{t}{T}\right)^{a_{0}}+G t^{a_{0}} \frac{1}{\left(a_{0}-\mu\right) t^{a_{0}-\mu}}=M\left(\frac{t}{T}\right)^{a_{0}}+\frac{G}{\left(a_{0}-\mu\right)} t^{\mu}
\end{aligned}
$$

on $\mathcal{D}$. We can get the estimate of $|(\partial w / \partial x)(t, x)|$ in the same way.

\subsection{Proof of Theorem 4.1}

Suppose (3.1), (3.2) and $0<\mu \leq \operatorname{Re} \lambda(0,0)$. We set $N=[\operatorname{Re} \lambda(0,0) / \mu]$, the integer part of $\operatorname{Re} \lambda(0,0) / \mu$; then we have $N \mu \leq \operatorname{Re} \lambda(0,0)<(N+1) \mu$.

If $N \mu<\operatorname{Re} \lambda(0,0)$, we set $d=\mu$. If $N \mu=\operatorname{Re} \lambda(0,0)$, we take $0<d<\mu$ so that $N d<\operatorname{Re} \lambda(0,0)<(N+1) d$. Then, in any case we have

$$
0<d \leq \mu<2 d \quad \text { and } \quad N d<\operatorname{Re} \lambda(0,0)<(N+1) d .
$$

After that, we take $0<T^{*}<T_{0}, 0<R^{*}<R_{0}$ and $a_{0}<a_{1}$ so that

$$
N d<a_{0} \leq \operatorname{Re} \lambda(t, x) \leq a_{1}<(N+1) d \quad \text { on }\left[0, T^{*}\right] \times D_{R^{*}} .
$$

Under this situation, let us look for a solution of (2.1) of the form

$$
u(t, x)=v(t, x)+w(t, x) \quad \text { with } \quad v(t, x)=\sum_{n=1}^{N} u_{n}(t, x) .
$$

Actually, we define $u_{n}(t, x)(n=1, \ldots, N)$ and $w(t, x)$ by the following recurrence formulas:

$$
L u_{1}=a(t, x),
$$

for $2 \leq n \leq N$

$$
L u_{n}=\sum_{2 \leq j+\alpha \leq n} a_{j, \alpha}(t, x) \sum_{|\vec{k}|+|\vec{m}|=n} u_{k_{1}} \cdots u_{k_{j}} \frac{\partial u_{m_{1}}}{\partial x} \cdots \frac{\partial u_{m_{\alpha}}}{\partial x}
$$


(where $\vec{k}=\left(k_{1}, \ldots, k_{j}\right),|\vec{k}|=k_{1}+\cdots+k_{j}, \vec{m}=\left(m_{1}, \ldots, m_{\alpha}\right)$ and $|\vec{m}|=$ $\left.m_{1}+\cdots+m_{\alpha}\right)$, and

$$
\begin{aligned}
L w= & R\left(t, x, v+w, \frac{\partial v}{\partial x}+\frac{\partial w}{\partial x}\right)-R\left(t, x, v, \frac{\partial v}{\partial x}\right) \\
& +\sum_{j+\alpha \geq 2} a_{j, \alpha}(t, x) \sum_{|\vec{k}|+|\vec{m}| \geq N+1} u_{k_{1}} \cdots u_{k_{j}} \frac{\partial u_{m_{1}}}{\partial x} \cdots \frac{\partial u_{m_{\alpha}}}{\partial x} .
\end{aligned}
$$

Step 1: By applying Proposition 4.2 (and Corollary 4.3) to (4.4) we have a solution $u_{1}(t, x) \in X_{1}^{d}\left(\left[0, T_{1}\right] \times D_{R_{1}}\right)$ for some $0<T_{1}<T^{*}$ and $0<R_{1}<$ $R^{*}$. Then, the right side of (4.5) (with $\left.n=2\right)$ belongs to $X_{1}^{2 d}\left(\left[0, T_{1}\right] \times D_{R_{1}}\right)$ and so in the case $2 \leq N$ we have a solution $u_{2}(t, x) \in X_{1}^{2 d}\left(\left[0, T_{2}\right] \times D_{R_{2}}\right)$ for some $0<T_{2}<T_{1}$ and $0<R_{2}<R_{1}$.

By repeating the same argument we have $0<T_{N}<\cdots<T_{2}<T_{1}$, $0<R_{N}<\cdots<R_{2}<R_{1}$ and $u_{n}(t, x) \in X_{1}^{n d}\left(\left[0, T_{n}\right] \times D_{R_{n}}\right)(1 \leq n \leq N)$ so that they satisfy the recurrence formulas (4.4) and (4.5) $(2 \leq n \leq N)$.

Step 2: Now, let us solve (4.6). We set

$$
f(t, x)=\sum_{j+\alpha \geq 2} a_{j, \alpha}(t, x) \sum_{|\vec{k}|+|\vec{m}| \geq N+1} u_{k_{1}} \cdots u_{k_{j}} \frac{\partial u_{m_{1}}}{\partial x} \cdots \frac{\partial u_{m_{\alpha}}}{\partial x}:
$$

we have the conditions $f(t, x) \in X_{0}\left(\left[0, T_{N}\right] \times D_{R_{N}}\right)$ and

$$
|f(t, x)| \leq F t^{(N+1) d} \quad \text { on }\left[0, T_{N}\right] \times D_{R_{N}}
$$

for some $F>0$. Since $v(t, x)$ is a known function in $X_{1}^{d}\left(\left[0, T_{N}\right] \times D_{R_{N}}\right)$, we have the expression

$$
\begin{aligned}
& R\left(t, x, v+w, \frac{\partial v}{\partial x}+\frac{\partial w}{\partial x}\right)-R\left(t, x, v, \frac{\partial v}{\partial x}\right) \\
& =c_{1}(t, x) w+c_{2}(t, x) \frac{\partial w}{\partial x}+\sum_{j+\alpha \geq 2} c_{j, \alpha}(t, x) w^{j}\left(\frac{\partial w}{\partial x}\right)^{\alpha},
\end{aligned}
$$

where $c_{i}(t, x) \in X_{0}\left(\left[0, T_{N}\right] \times D_{R_{N}}\right)(i=1,2), c_{j, \alpha}(t, x) \in X_{0}\left(\left[0, T_{N}\right] \times D_{R_{N}}\right)$ $(j+\alpha \geq 2)$, and we have

$$
\left|c_{i}(t, x)\right| \leq C_{i} t^{d} \quad \text { on }\left[0, T_{N}\right] \times D_{R_{N}}
$$

for some $C_{i}>0(i=1,2)$.

Hence, under the setting

$$
\begin{aligned}
& \lambda_{c}(t, x)=\lambda(t, x)+c_{1}(t, x) \\
& b_{c}(t, x)=b(t, x)+c_{2}(t, x), \\
& L_{c}=t \frac{\partial}{\partial t}-\lambda_{c}(t, x)-b_{c}(t, x) \frac{\partial}{\partial x}
\end{aligned}
$$


our equation (4.6) is written in the form

$$
L_{c} w=f(t, x)+\sum_{j+\alpha \geq 2} c_{j, \alpha}(t, x) w^{j}\left(\frac{\partial w}{\partial x}\right)^{\alpha} .
$$

Since (4.8) is valid, we have

$$
\begin{aligned}
& \operatorname{Re} \lambda_{c}(0,0)=\operatorname{Re} \lambda(0,0)<a_{1}, \\
& \left|b_{c}(t, x)\right| \leq\left(B+C_{2}\right)\left(\mu(t)+t^{d}\right) \quad \text { on }\left[0, T_{N}\right] \times D_{R_{N}} .
\end{aligned}
$$

Since (4.7) is satisfied and since $(N+1) d>a_{1}$ holds, we can apply Theorem 3.1 to (4.9) and we obtain a solution $w(t, x) \in X_{1}^{((N+1) d)}$.

Step 3: Thus, by setting

$$
u(t, x)=\sum_{n=1}^{N} u_{n}(t, x)+w(t, x)
$$

we have a solution $u(t, x)$ of (2.1) belonging to $X_{1}^{d}$.

Now, let us recall the construction of $u_{1}(t, x)$ : it is a solution of (4.4). Since (3.1) is supposed, by Corollary 4.3 we have $u_{1}(t, x) \in X_{1}^{\mu}$ if $\mu<$ $\operatorname{Re} \lambda(0,0)$ (resp. $u_{1}(t, x) \in X_{1}^{(\mu)}$ if $\left.\mu=\operatorname{Re} \lambda(0,0)\right)$. Hence, we have $u(t, x) \in$ $X_{1}^{\mu}$ if $\mu<\operatorname{Re} \lambda(0,0)$ (resp. $u(t, x) \in X_{1}^{(\mu)}$ if $\mu=\operatorname{Re} \lambda(0,0)$ ). This completes the proof of Theorem 4.1 .

\section{Reduction of the problem}

By Theorem 3.1 and Theorem 4.1 we have the result (1) of Theorem 2.3 . Take any $u_{0}(t, x) \in \mathcal{S}\left((2.1), X_{1}^{(\mu)}\right)$ and fix it.

Now, let $u(t, x)$ be a solution of (2.1). Set

$$
U(t, x)=u(t, x)-u_{0}(t, x):
$$

then, equation (2.1) is transformed to an equation with respect to $U(t, x)$ :

$$
\begin{aligned}
t \frac{\partial U}{\partial t}= & \lambda(t, x) U+b(t, x) \frac{\partial U}{\partial x} \\
& +R\left(t, x, u_{0}+U, \frac{\partial u_{0}}{\partial x}+\frac{\partial U}{\partial x}\right)-R\left(t, x, u_{0}, \frac{\partial u_{0}}{\partial x}\right) .
\end{aligned}
$$

Hence, to show the results (2) and (3) of Theorem 2.3 it is sufficient to prove the following theorem.

Theorem 5.1. Suppose the conditions $\left.\left.\mathrm{A}_{1}\right) \sim \mathrm{A}_{3}\right)$, (1.3), and $\left.\left.\mathrm{c} 1\right) \sim \mathrm{c} 3\right)$. Then, we have the following results. 
(1) For any $\psi(x) \in \mathcal{O}_{0}$ the equation (5.1) has a unique solution $U(t, x) \in$ $X_{1}^{+}$satisfying

$$
\lim _{t \rightarrow+0}\left(t^{-\lambda(0, x)} U(t, x)\right)=\psi(x) \quad \text { uniformly near } x=0 .
$$

From now, we write this solution as $U(\psi)(t, x)$.

(2) We denote by $\mathcal{S}\left(\right.$ (5.1),$X$ ) (where $X=\mathscr{X}_{1}$ or $X=X_{1}^{+}$) the set of all solutions of (5.1) belonging to $X$. We have

$$
\mathcal{S}\left(\text { (5.1) }, \mathscr{X}_{1}\right)=\mathcal{S}\left(\text { (5.1) }, X_{1}^{+}\right)=\left\{U(\psi) ; \psi(x) \in \mathcal{O}_{0}\right\} .
$$

The rest part of this paper will be used to prove Theorem 5.1. Take any $0<d<\mu$. Since $u_{0}(t, x)$ is a known function belonging to $X_{1}^{d}$, we have the expression

$$
\begin{aligned}
& R\left(t, x, u_{0}+U, \frac{\partial u_{0}}{\partial x}+\frac{\partial U}{\partial x}\right)-R\left(t, x, u_{0}, \frac{\partial u_{0}}{\partial x}\right) \\
& =c_{1}(t, x) U+c_{2}(t, x) \frac{\partial U}{\partial x}+\sum_{j+\alpha \geq 2} c_{j, \alpha}(t, x) U^{j}\left(\frac{\partial U}{\partial x}\right)^{\alpha}
\end{aligned}
$$

where $c_{i}(t, x) \in X_{0}\left([0, T] \times D_{R}\right)(i=1,2), c_{j, \alpha}(t, x) \in X_{0}\left([0, T] \times D_{R}\right)$ $(j+\alpha \geq 2)$ for some $T>0$ and $R>0$, and we have $\left|c_{i}(t, x)\right| \leq C_{i} t^{d}$ on $[0, T] \times D_{R}$ for some $C_{i}>0(i=1,2)$. Then, our equation (5.1) is written in the form

$$
t \frac{\partial U}{\partial t}=\lambda_{c}(t, x) U+b_{c}(t, x) \frac{\partial U}{\partial x}+\sum_{j+\alpha \geq 2} c_{j, \alpha}(t, x) U^{j}\left(\frac{\partial U}{\partial x}\right)^{\alpha},
$$

where

$$
\begin{aligned}
& \lambda_{c}(t, x)=\lambda(t, x)+c_{1}(t, x), \\
& b_{c}(t, x)=b(t, x)+c_{2}(t, x) .
\end{aligned}
$$

Without loss of generality, we may asuume: $0<T<1 / e$. By (1.3), c2) and c3) we have

$$
\begin{aligned}
& \operatorname{Re} \lambda_{c}(0,0)=\operatorname{Re} \lambda(0,0)>0 \\
& \left|\lambda_{c}(t, x)-\lambda_{c}(0, x)\right| \leq|\lambda(t, x)-\lambda(0, x)|+\left|c_{1}(t, x)\right| \\
& \quad \leq \Lambda_{1}\left(\mu(t)+t^{d}\right) \leq \Lambda_{1}\left(\mu(t)+|\log t| t^{d}\right), \\
& \left|b_{c}(t, x)\right| \leq|b(t, x)|+\left|c_{2}(t, x)\right| \leq \frac{B_{1}\left(\mu(t)+|\log t| t^{d}\right)}{|\log t|}
\end{aligned}
$$

on $[0, T] \times D_{R}$ for some $\Lambda_{1}>0$ and $B_{1}>0$. Since $\mu_{0}(t)=\mu(t)+|\log t| t^{d}$ is also a weight function on $(0, T]$, the equation (15.2) satisfies similar conditions as (1.3), c2) and c3): the only difference is that we have $a(t, x) \equiv 0$ in the case (5.2).

Thus, for the sake of simplicity of notations, instead of (5.2) we may treat (2.1) under the condition $a(t, x) \equiv 0$. 


\section{Analysis in the case $a(t, x) \equiv 0$}

In this section, we consider the equation (2.1) in the case $a(t, x) \equiv 0$; then our equation is

$$
t \frac{\partial u}{\partial t}=\lambda(t, x) u+b(t, x) \frac{\partial u}{\partial x}+R\left(t, x, u, \frac{\partial u}{\partial x}\right)
$$

which has a trivial solution $u(t, x) \equiv 0$.

\subsection{On a basic equation $L v=0$}

First, let

$$
L=t \frac{\partial}{\partial t}-\lambda(t, x)-b(t, x) \frac{\partial}{\partial x}
$$

be as before, and let us consider the equation

$$
L v=0
$$

in the case $\lambda(0, x) \equiv 0$ on $D_{R_{0}}$, under the conditions: $\lambda(t, x), b(t, x) \in$ $X_{0}\left(\left[0, T_{0}\right] \times D_{R_{0}}\right)$ and

$$
\begin{array}{ll}
|\lambda(t, x)| \leq \Lambda \mu(t) & \text { on }\left[0, T_{0}\right] \times D_{R_{0}}, \\
|b(t, x)| \leq B \mu(t) & \text { on }\left[0, T_{0}\right] \times D_{R_{0}}
\end{array}
$$

for some $\Lambda>0, B>0$ and a weight function $\mu(t)$ on $\left(0, T_{0}\right]$.

We take $0<T<T_{0}, 0<R<R_{0}, 0<r \leq 1 / B$ and set

$$
W=\left\{(t, x) \in[0, T] \times D_{R} ; \varphi(t) / r+|x|<R\right\} .
$$

Let $\phi\left(t ; t_{0}, x_{0}\right)$ be the unique solution of (4.2) as before. For $x_{0} \in D_{R}$ we set

$$
t_{x_{0}}=\sup \left\{t_{1}>0 ;\left(t, \phi\left(t ; 0, x_{0}\right)\right) \in W \text { for any } 0 \leq t<t_{1}\right\} .
$$

Lemma 6.1.1. (1) For any $\left(t_{0}, x_{0}\right) \in W$, we have $\left(t, \phi\left(t ; t_{0}, x_{0}\right)\right) \in W$ for any $0 \leq t \leq t_{0}$.

(2) We have

$$
W=\bigcup_{x_{0} \in D_{R}}\left\{\left(t, \phi\left(t ; 0, x_{0}\right)\right) ; 0 \leq t<t_{x_{0}}\right\} .
$$

Proof. Set $x(t)=\phi\left(t ; t_{0}, x_{0}\right)$ : then we have

$$
x(t)=x_{0}+\int_{t}^{t_{0}} \frac{b(\tau, x(\tau))}{\tau} d \tau .
$$

If $x(t)$ exists on $\left(t_{1}, t_{0}\right]$, for any $t \in\left(t_{1}, t_{0}\right]$ we have

$$
|x(t)| \leq\left|x_{0}\right|+B \int_{t}^{t_{0}} \frac{\mu(\tau)}{\tau} d \tau=\left|x_{0}\right|+B\left(\varphi\left(t_{0}\right)-\varphi(t)\right)
$$


and so by the condition $1 / r \geq B$ we have

$$
\begin{aligned}
\varphi(t) / r+|x(t)| & \leq \varphi(t) / r+\left|x_{0}\right|+B\left(\varphi\left(t_{0}\right)-\varphi(t)\right) \\
& =\varphi\left(t_{0}\right) / r+\left|x_{0}\right|-(1 / r-B)\left(\varphi\left(t_{0}\right)-\varphi(t)\right) \\
& \leq \varphi\left(t_{0}\right) / r+\left|x_{0}\right|<R .
\end{aligned}
$$

This shows that $x(t)$ can be extended to $\left[0, t_{0}\right]$. This proves (1). The result (2) follows from (1).

As to (6.1.1) we have

Lemma 6.1.2. Suppose the conditions (6.1.2) and (6.1.3). Then, we have the following results.

(1) For any holomorphic function $\psi(x)$ on $D_{R}$ the equation (6.1.1) has a unique solution $v(t, x) \in X_{1}(W)$ satisfying $v(0, x)=\psi(x)$ on $D_{R}$, and it is given by

$$
v(t, x)=\psi(\phi(0 ; t, x)) \exp \left[\int_{0}^{t} \frac{\lambda(s, \phi(s ; t, x))}{s} d s\right] .
$$

We write this solution as $V_{0}(\psi)(t, x)$.

(2) If $v(t, x) \in X_{1}\left(\left(0, T_{1}\right] \times D_{R_{1}}\right)$ is a solution of (6.1.1) we have $v(t, x) \in$ $X_{1}\left([0, T] \times D_{R}\right)$ for some $T>0$ and $R>0$. By setting $\psi(x)=v(0, x)$ we have $v=V_{0}(\psi)$ on $[0, \epsilon] \times D_{\delta}$ for some $\psi(x) \in \mathcal{O}_{0}, \epsilon>0$ and $\delta>0$.

Proof. First, let us show (1). Let $v(t, x) \in X_{1}(W)$ be a solution of (6.1.1) satisfying $v(0, x)=\psi(x)$ on $D_{R}$. Take any $x_{0} \in D_{R}$. Set

$$
\begin{aligned}
& v^{*}(t)=v\left(t, \phi\left(t ; 0, x_{0}\right)\right), \\
& \lambda^{*}(t)=\lambda\left(t, \phi\left(t ; 0, x_{0}\right)\right) .
\end{aligned}
$$

Then, our equation (6.1.1) is written in the form

$$
t \frac{d v^{*}}{d t}-\lambda^{*}(t) v^{*}=0, \quad v^{*}(0)=\psi\left(x_{0}\right) .
$$

By integrating this, we have

$$
v^{*}(t)=\psi\left(x_{0}\right) \exp \left[\int_{0}^{t} \frac{\lambda^{*}(s)}{s} d s\right]
$$

that is,

$$
v\left(t, \phi\left(t ; 0, x_{0}\right)\right)=\psi\left(x_{0}\right) \exp \left[\int_{0}^{t} \frac{\lambda\left(s, \phi\left(s ; 0, x_{0}\right)\right)}{s} d s\right] .
$$

Take any $(t, x) \in W$; by Lemma 6.1.1 we have a unique $x_{0} \in D_{R}$ such that $x=\phi\left(t ; 0, x_{0}\right)$. In this case, we have $x_{0}=\phi(0 ; t, x)$ and $\phi\left(s ; 0, x_{0}\right)=$ 
$\phi(s ; t, x)$ for $0 \leq s \leq t$. Therefore, by replacing $x_{0}$ and $\phi\left(s ; 0, x_{0}\right)$ by $\phi(0 ; t, x)$ and $\phi(s ; t, x)$, respectively, in the above formula we finally obtain

$$
v(t, x)=\psi(\phi(0 ; t, x)) \exp \left[\int_{0}^{t} \frac{\lambda(s, \phi(s ; t, x))}{s} d s\right] .
$$

This proves the formula (6.1.4). This shows also the uniqueness of the solution.

To show the existence of a solution, it is enough to see that $v(t, x)$ defined by (6.1.4) is a desired solution. Since (6.1.2) is supposed, we have the welldefinedness of $v(t, x)$ as a function in $X_{1}(W)$ and $v(0, x)=\psi(x)$ on $D_{R}$. Thus, the result (1) is proved.

Next, let us show (2). Let $v(t, x) \in X_{1}\left(\left(0, T_{1}\right] \times D_{R_{1}}\right)$ be a solution of (6.1.1). Take $0<T<T_{1}$ and $0<R<R_{2}<R_{1}$ so that

$$
R+B \varphi(T)<R_{2}, \quad R_{2}+B \varphi(T)<R_{1} .
$$

Set

$$
\mathcal{D}=\bigcup_{x_{0} \in D_{R_{2}}}\left\{\left(t, \phi\left(t ; T, x_{0}\right)\right) ; 0<t \leq T\right\} .
$$

It is easy to see that $(0, T] \times D_{R} \subset \mathcal{D} \subset(0, T] \times D_{R_{1}}$ and that $\mathcal{D} \cap\left\{t=t_{0}\right\}$ is an open subset of $\mathbb{C}$ for any $t_{0} \in(0, T]$. By Proposition 4.2 we have the expression

$$
v(t, x)=v\left(T,(\phi(T ; t, x)) \exp \left[-\int_{t}^{T} \frac{\lambda(s, \phi(s ; t, x))}{s} d s\right] \quad \text { on } \mathcal{D} .\right.
$$

Since (6.1.2) is supposed, by using this expression we can see that

$$
\sup _{x \in D_{R}}\left|v\left(t_{1}, x\right)-v\left(t_{2}, x\right)\right| \longrightarrow 0 \quad\left(\text { as } t_{1}, t_{2} \longrightarrow+0\right) .
$$

This concludes that $v(t, x)$ converges to a holomorphic function $\psi(x)$ (as $t \longrightarrow+0)$ uniformly on $D_{R}$. Hence, by setting $v(0, x)=\psi(x)$ we have the condition $v(t, x) \in X_{1}\left([0, T] \times D_{R}\right)$. This proves the former half of $(2)$. The latter half of (2) follows from the uniqueness of the solution in (1).

\subsection{Singular Cauchy problem for (6.1.1)}

Let us consider the same equation (6.1.1) in the case $\lambda(0, x) \not \equiv 0$ on $D_{R_{0}}$. In this case, instead of the Cauchy data $v(0, x)=\psi(x)$ we impose the following singular Cauchy data:

$$
\lim _{t \rightarrow+0} t^{-\lambda(0, x)} v(t, x)=\psi(x)
$$


To do so, instead of (6.1.2) and (6.1.3) we suppose: $0<T_{0}<1 / e$,

$$
\begin{aligned}
& |\lambda(t, x)-\lambda(0, x)| \leq \Lambda \mu(t) \quad \text { on }\left[0, T_{0}\right] \times D_{R_{0}}, \\
& |b(t, x)| \leq \frac{B \mu(t)}{|\log t|} \quad \text { on }\left[0, T_{0}\right] \times D_{R_{0}}
\end{aligned}
$$

for some $\Lambda>0, B>0$ and a weight function $\mu(t)$ on $\left(0, T_{0}\right]$. We note that (6.2.2) implies (6.1.3).

For $T>0, R>0$ and $r>0$ we set $W=\left\{(t, x) \in[0, T] \times D_{R} ; \varphi(t) / r+\right.$ $|x|<R\}$, as before.

Lemma 6.2.1. Suppose (6.2.1), (6.2.2) and $\operatorname{Re} \lambda(0,0)>0$. Then, we have the following results.

(1) There are $T>0, R>0$ and $r>0$ which satisfy the following: for any holomorphic function $\psi(x)$ on $D_{R}$ the equation (6.1.1) has a unique solution $v(t, x) \in X_{1}(W)$ satisfying

$$
t^{-\lambda(0, x)} v(t, x) \longrightarrow \psi(x) \quad(\text { as } t \longrightarrow+0)
$$

uniformly on $D_{R^{\prime}}$ for any $0<R^{\prime}<R$. In addition, we have $v(t, x) \in X_{1}^{d}$ for any $0<d<\operatorname{Re} \lambda(0,0)$. We write this solution as $V(\psi)(t, x)$.

(2) If $v(t, x) \in X_{1}\left(\left(0, T_{1}\right] \times D_{R_{1}}\right)$ is a solution of (6.1.1), we have $v=$ $V(\psi)$ on $(0, \epsilon] \times D_{\delta}$ for some $\psi(x) \in \mathcal{O}_{0}, \epsilon>0$ and $\delta>0$.

Proof. We set $w(t, x)=t^{-\lambda(0, x)} v(t, x)$. Then, (6.1.1) is reduced to an equation with respecto to $w(t, x)$ :

$$
t \frac{\partial w}{\partial t}=\lambda_{b}(t, x) w+b(t, x) \frac{\partial w}{\partial x}
$$

where

$$
\lambda_{b}(t, x)=(\lambda(t, x)-\lambda(0, x))+\frac{\partial \lambda(0, x)}{\partial x}(\log t) b(t, x) .
$$

Since $0<T_{0}<1 / e$, (6.2.1) and (6.2.2) are supposed, we have

$$
\begin{aligned}
& \left|\lambda_{b}(t, x)\right| \leq \Lambda \mu(t)+C B \mu(t) \quad \text { on }\left[0, T_{0}\right] \times D_{R_{1}}, \\
& |b(t, x)| \leq B \mu(t) /|\log t| \leq M \mu(t) \quad \text { on }\left[0, T_{0}\right] \times D_{R_{1}} .
\end{aligned}
$$

for some $C>0$ and $0<R_{1}<R_{0}$. Hence, we can apply Lemma 6.1.2 to (6.2.3). This leads us to Lemma 6.2.1.

\subsection{Singular Cauchy problem for (6.0.1)}

Let us consider the singular Cauchy problem for (6.0.1). We have 
Theorem 6.3.1. Suppose (6.2.1), (6.2.2) and $\operatorname{Re} \lambda(0,0)>0$. Then, for any $\psi(x) \in \mathcal{O}_{0}$ the equation (6.0.1) has a unique solution $u(t, x) \in X_{1}^{+}$ satiasfying

$$
t^{-\lambda(0, x)} u(t, x) \longrightarrow \psi(x) \quad(\text { as } t \longrightarrow+0)
$$

uniformly on $D_{R}$ for some $R>0$. We write this solution as $U(\psi)(t, x)$.

Proof. We take $0<T_{1}<T_{0}, 0<R_{1}<R_{0}, d>0, a_{1}>a_{0}>0$ so that

$$
0<d<a_{0} \leq \operatorname{Re} \lambda(t, x) \leq a_{1}<2 d \quad \text { on }\left[0, T_{1}\right] \times D_{R_{1}} .
$$

Step 1: Let us look for a solution $u(t, x)$ in the form

$$
u(t, x)=v(t, x)+w(t, x)
$$

where $v(t, x)$ and $w(t, x)$ are defined by the recurrence formulas:

$$
\begin{aligned}
& L v=0, \\
& L w=R\left(t, x, v+w, \frac{\partial v}{\partial x}+\frac{\partial w}{\partial x}\right) .
\end{aligned}
$$

By Lemma 6.2.1 we have a solution $v(t, x) \in X_{1}^{d}\left(\left[0, T_{2}\right] \times D_{R_{2}}\right)$ of (6.3.3) (for some $0<T_{2}<T_{1}$ and $0<R_{2}<R_{1}$ ) satisfying

$$
t^{-\lambda(0, x)} v(t, x) \longrightarrow \psi(x) \quad(\text { as } t \longrightarrow+0)
$$

uniformly on $D_{R_{2}}$. Then, $v(t, x)$ is a known function, and under the setting

$$
f(t, x)=R\left(t, x, v, \frac{\partial v}{\partial x}\right)
$$

our equation (6.3.4) is written in the form

$$
L w=f(t, x)+R\left(t, x, v+w, \frac{\partial v}{\partial x}+\frac{\partial w}{\partial x}\right)-R\left(t, x, v, \frac{\partial v}{\partial x}\right) .
$$

Since $v(t, x) \in X_{1}^{d}\left(\left[0, T_{2}\right] \times D_{R_{2}}\right)$ is valid, we have

$$
|f(t, x)| \leq F t^{2 d} \quad \text { on }\left[0, T_{2}\right] \times D_{R_{2}}
$$

for some $F>0$. Since $2 d>a_{1}$ is supposed in (6.3.2), by the same argument as in the proof of Theorem 4.1 (in Step 2 in subsection 4.2) we have a solution $w(t, x) \in X_{1}^{(2 d)}$ of (6.3.5). Since $2 d>a_{1}$ holds, we have

$$
t^{-\lambda(0, x)} w(t, x) \longrightarrow 0 \quad(\text { as } t \longrightarrow+0) .
$$

Thus, by setting $u(t, x)=v(t, x)+w(t, x)$ we have a solution of (6.0.1) satisfying (6.3.1). This proves the existence part of Theorem 6.3.1. 
Step 2: Next, let us show the uniqueness of the solution. Let $u_{i}(t, x) \in$ $X_{1}^{+}(i=1,2)$ be two solutions of (6.0.1) satisfying (6.3.1). By (6.3.1) and (6.3.2) we have $u_{i}(t, x) \in X_{1}^{d}(i=1,2)$. Set $w(t, x)=u_{1}(t, x)-u_{2}(t, x) \in X_{1}^{d}$. Then, we have

$$
L w=R\left(t, x, u_{2}+w, \frac{\partial u_{2}}{\partial x}+\frac{\partial w}{\partial x}\right)-R\left(t, x, u_{2}, \frac{\partial u_{2}}{\partial x}\right)
$$

and $t^{-\lambda(0, x)} w(t, x) \longrightarrow 0$ (as $t \longrightarrow+0$ ) uniformly near $x=0$. Since $u_{2}(t, x)$ and $w(t, x)$ are known function, we have the expression

$$
R\left(t, x, u_{2}+w, \frac{\partial u_{2}}{\partial x}+\frac{\partial w}{\partial x}\right)-R\left(t, x, u_{2}, \frac{\partial u_{2}}{\partial x}\right)=h_{1}(t, x) w+h_{2}(t, x) \frac{\partial w}{\partial x}
$$

for some $h_{i}(t, x) \in X_{0}\left([0, T] \times D_{R}\right)$ (with $T>0, R>0$ ), and we have

$$
\left|h_{i}(t, x)\right| \leq H_{i} t^{d} \quad \text { on }[0, T] \times D_{R}
$$

for some $H_{i}>0(i=1,2)$. Then, under the setting

$$
L_{h}=t \frac{\partial}{\partial t}-\left(\lambda(t, x)+h_{1}(t, x)\right) w-\left(b(t, x)+h_{2}(t, x)\right) \frac{\partial}{\partial x}
$$

we have

$$
L_{h} w=0, \quad t^{-\lambda(0, x)} w(t, x) \longrightarrow 0 \quad(\text { as } t \longrightarrow+0) .
$$

By applying the uniqueness part of Lemma 6.2.1 (with $L$ and $\mu(t)$ replaced by $L_{h}$ and $\left.\mu_{0}(t)=\mu(t)+|\log t| t^{d}\right)$ to (6.3.6) we have $w(t, x)=0$ on $[0, \epsilon] \times D_{\delta}$ for some $\epsilon>0$ and $\delta>0$. Hence, we have $u_{1}(t, x)=u_{2}(t, x)$ on $[0, \epsilon] \times D_{\delta}$.

This proves the uniqueness of the solution.

\subsection{On $\mathcal{S}\left((\underline{6.0 .1)}), X_{1}^{+}\right)$}

We denote by $\mathcal{S}\left(\right.$ (6.0.1),$\left.X_{1}^{+}\right)$the set of all solutions of (6.0.1) belonging to the class $X_{1}^{+}$. By Theorem 6.3 .1 we have

$$
\mathcal{S}\left((\underline{6.0 .1)}), X_{1}^{+}\right) \supset\left\{U(\psi) ; \psi(x) \in \mathcal{O}_{0}\right\} .
$$

Let us show

Theorem 6.4.1. Suppose (6.2.1), (6.2.2) and $\operatorname{Re} \lambda(0,0)>0$. Then, we have

$$
\mathcal{S}\left((6.0 .1), X_{1}^{+}\right)=\left\{U(\psi) ; \psi(x) \in \mathcal{O}_{0}\right\} .
$$

Proof. Take any $u(t, x) \in \mathcal{S}\left(\right.$ (6.0.1),$\left.X_{1}^{+}\right)$. We have $u(t, x) \in X_{1}^{\mu}\left(\left[0, T_{1}\right] \times\right.$ $D_{R_{1}}$ ) for some $\mu>0, T_{1}>0$ and $R_{1}>0$. Without loss of generality, we may assume that $0<\mu<\operatorname{Re} \lambda(0,0)$ holds.

1) If $\operatorname{Re} \lambda(0,0)<2 \mu$, we set $d_{1}=\mu$ and $N=1$. 
2) If $2 \mu \leq \operatorname{Re} \lambda(0,0)$, we take $d_{i}>0(i=1, \ldots, N)$ so that

$$
0<d_{1}<\mu<d_{2}<\cdots<d_{N}<\operatorname{Re} \lambda(0,0)<2 d_{N}
$$

and that $d_{i+1} \leq 2 d_{i}(i=1, \ldots, N-1)$ hold.

Step 1: In the case 2) we have $u(t, x) \in X_{1}^{d_{2}}$. The proof is as follows.

By taking $a_{0}>0, T_{1}>0$ and $R_{1}>0$ suitably, we have the condition

$$
\mu<d_{2}<a_{0} \leq \operatorname{Re} \lambda(t, x) \text { on }\left[0, T_{1}\right] \times D_{R_{1}} .
$$

We set

$$
f(t, x)=R\left(t, x, u, \frac{\partial u}{\partial x}\right):
$$

we have $f(t, x) \in X_{0}\left(\left[0, T_{1}\right] \times D_{R_{1}}\right)$. Since $u \in X_{1}^{\mu}\left(\left[0, T_{1}\right] \times D_{R_{1}}\right)$ and $d_{2}<2 \mu$ hold, we have the estimate $|f(t, x)| \leq F t^{d_{2}}$ on $\left[0, T_{1}\right] \times D_{R_{1}}$ for some $F>0$. By applying Proposition 4.2 (and Corollary 4.3) to $L w=f(t, x)$ we have a solution $w(t, x) \in X_{1}^{d_{2}}$ of $L w=f(t, x)$. Since $u(t, x)$ is a solution of $L u=f(t, x)$, by setting $v(t, x)=u(t, x)-w(t, x)$ we have an equation

$$
L v=0
$$

and $v(t, x) \in X_{1}^{\mu}$. Then, by (2) of Lemma 6.2.1 we have an expression $v=V(\psi)$ for some $\psi(x) \in \mathcal{O}_{0}$, that is, we have the condition:

$$
t^{-\lambda(0, x)} v(t, x) \longrightarrow \psi(x) \quad(\text { as } t \longrightarrow+0)
$$

uniformly on $D_{R}$ for some $R>0$. This means that $v \in X_{1}^{\left(a_{0}\right)}$. Hence, we have

$$
u(t, x)=v(t, x)+w(t, x) \in X_{1}^{\left(a_{0}\right)}+X_{1}^{d_{2}} \subset X_{1}^{d_{2}} .
$$

Step 2: In the case 1) we know $u(t, x) \in X_{1}^{d_{N}}$ from the first. In the case 2 ), by Step 1 we have $u(t, x) \in X_{1}^{d_{2}}$ : then, by applying the same argument we have $u(t, x) \in X_{1}^{d_{3}}$, and so by repeating the same argument we have finally the condition $u(t, x) \in X_{1}^{d_{N}}$. Thus, in any case we have $u(t, x) \in X_{1}^{d_{N}}$.

Then, the function $f(t, x)$ in (6.4.2) satisfies $|f(t, x)| \leq F_{1} t^{2 d_{N}}$ on $\left[0, T_{2}\right] \times$ $D_{R_{2}}$ for some $T_{2}>0$ and $R_{2}>0$. Since $2 d_{N}>\operatorname{Re} \lambda(0,0)$ holds, by applying Theorem 3.1 to $L w=f$ we have a solution $w(t, x) \in X_{1}^{\left(2 d_{N}\right)}$ of $L w=f$. Since $u(t, x)$ is a solution of $L u=f(t, x)$, by setting $v(t, x)=u(t, x)-w(t, x)$ we have an equation

$$
L v=0
$$

and $v(t, x) \in X_{1}^{d_{N}}$. Hence, by (2) of Lemma 6.2.1 we have an expression $v=V(\psi)$ for some $\psi(x) \in \mathcal{O}_{0}$, that is, we have the condition:

$$
t^{-\lambda(0, x)} v(t, x) \longrightarrow \psi(x) \quad(\text { as } t \longrightarrow+0)
$$


uniformly on $D_{R}$ for some $R>0$. Since $w(t, x) \in X_{1}^{\left(2 d_{N}\right)}$ and $2 d_{N}>$ $\operatorname{Re} \lambda(0,0)$ hold, we have $t^{-\lambda(0, x)} w(t, x) \longrightarrow 0$ (as $t \longrightarrow+0$ ) uniformly near $x=0$. Since $u(t, x)=v(t, x)+w(t, x)$, we obtain

$$
t^{-\lambda(0, x)} u(t, x) \longrightarrow \psi(x) \quad(\text { as } t \longrightarrow+0)
$$

uniformly near $x=0$. Thus, by the uniqueness of the solution in Theorem 6.3 .1 we have $u(t, x)=U(\psi)(t, x)$ on $[0, \epsilon] \times D_{\delta}$ for some $\epsilon>0$ and $\delta>0$.

This proves (6.4.1).

\subsection{On $\left.\mathcal{S}(6.0 .1), \mathscr{X}_{1}\right)$}

Lastly, we consider (6.0.1) under the conditions (6.1.3) and $\operatorname{Re} \lambda(0,0)>0$. The purpose is to prove

Theorem 6.5.1. Under the conditions (6.1.3) and $\operatorname{Re} \lambda(0,0)>0$, we have

$$
\mathcal{S}\left((6.0 .1), \mathscr{X}_{1}\right)=\mathcal{S}\left((6.0 .1), X_{1}^{+}\right) .
$$

Proof. Since $\mathcal{S}\left(\sqrt{6.0 .1)}, \mathscr{X}_{1}\right) \supset \mathcal{S}\left((6.0 .1), X_{1}^{+}\right)$is trivial, it is enough to prove that $\left.\left.\mathcal{S}(\sqrt[6.0 .1]{ }), \mathscr{X}_{1}\right) \subset \mathcal{S}(\sqrt{6.0 .1}), X_{1}^{+}\right)$holds. Let us show this from now.

The argument below is quite similar to the one used in the proof of [Theorem 2.2 in [13]], where we have treated the case $\operatorname{Re} \lambda(0,0)<0$. Since we are now considering the case $\operatorname{Re} \lambda(0,0)>0$, the treating of some parts must be changed.

Step 1: Take any $\left.u(t, x) \in \mathcal{S}(\underline{6.0 .1}), \mathscr{X}_{1}\right)$; then we have $u(t, x) \in X_{1}\left(\left(0, T_{1}\right] \times\right.$ $\left.D_{R_{1}}\right)$ and

$$
\varlimsup_{R \rightarrow+0}\left[\lim _{T \rightarrow+0}\left(\frac{1}{R^{2}} \sup _{(0, T] \times D_{R}}|u(t, x)|\right)\right]=0 .
$$

Since $u(t, x)$ is a solution of (6.0.1), under setting $q(t, x)=(\partial u / \partial x)(t, x)$ we have the relation

$$
t \frac{\partial u}{\partial t}=\lambda(t, x) u+b(t, x) \frac{\partial u}{\partial x}+R_{1}(t, x, u, q) u+R_{2}(t, x, u, q) \frac{\partial u}{\partial x}
$$

on $\left(0, T_{1}\right] \times D_{R_{1}}$ for some $R_{i}\left(t, x, z_{1}, z_{2}\right) \in C^{0}(\Omega)(i=1,2)$ that are holomorphic in the variable $\left(x, z_{1}, z_{2}\right) \in D_{R_{0}} \times D_{\rho_{0}} \times D_{\rho_{0}}$ for any fixed $t$ and that satisfy $R_{i}(t, x, 0,0) \equiv 0(i=1,2)$.

By taking $T_{1}>0$ and $R_{1}>0$ sufficiently small, we may assume that

$$
\begin{aligned}
& \operatorname{Re} \lambda(t, x)>2 a>0, \quad \text { on }\left[0, T_{1}\right] \times D_{R_{1}}, \\
& \left|R_{1}(t, x, u, q)\right| \leq A_{1}|u|+A_{2}|q|, \quad \text { on }\left[0, T_{1}\right] \times D_{R_{1}}, \\
& \left|R_{2}(t, x, u, q)\right| \leq B_{1}|u|+B_{2}|q|, \quad \text { on }\left[0, T_{1}\right] \times D_{R_{1}}
\end{aligned}
$$


for some $a>0, A_{1}>0, A_{2}>0, B_{1}>0$ and $B_{2}>0$. By setting

$$
\begin{aligned}
& \left.\lambda_{1}(t, x)=R_{1}(t, x, u(t, x), q(t . x)) \in X_{0}\left(\left(0, T_{1}\right] \times D_{R_{1}}\right)\right), \\
& \left.b_{1}(t, x)=R_{2}(t, x, u(t, x), q(t . x)) \in X_{0}\left(\left(0, T_{1}\right] \times D_{R_{1}}\right)\right)
\end{aligned}
$$

we have the relation

$$
t \frac{\partial u}{\partial t}-\left(b(t, x)+b_{1}(t, x)\right) \frac{\partial u}{\partial x}=\left(\lambda(t, x)+\lambda_{1}(t, x)\right) u
$$

on $\left(0, T_{1}\right] \times D_{R_{1}}$. By applying $\partial / \partial x$ to (6.5.3) we have

$$
\begin{aligned}
t \frac{\partial q}{\partial t}-\left(b(t, x)+b_{1}(t, x)\right) \frac{\partial q}{\partial x} & \\
& =\left(\lambda(t, x)+\lambda_{1}(t, x)+c(t, x)\right) q+\gamma(t, x) u
\end{aligned}
$$

on $\left(0, T_{1}\right] \times D_{R_{1}}$, where

$$
\begin{aligned}
& c(t, x)=\frac{\partial b}{\partial x}(t, x)+\frac{\partial b_{1}}{\partial x}(t, x) \in X_{0}\left(\left(0, T_{1}\right] \times D_{R_{1}}\right), \\
& \gamma(t, x)=\frac{\partial \lambda}{\partial x}(t, x)+\frac{\partial \lambda_{1}}{\partial x}(t, x) \in X_{0}\left(\left(0, T_{1}\right] \times D_{R_{1}}\right) .
\end{aligned}
$$

Step 2: For $0<T<T_{1}$ and $0<R<R_{1}$ we set

$$
\begin{aligned}
& r_{1}=\sup _{(0, T] \times D_{R}}|u(t, x)|, \quad r_{2}=\sup _{(0, T] \times D_{R}}|q(t, x)|, \\
& C=\sup _{(0, T] \times D_{R}}|c(t, x)|, \quad L=\sup _{(0, T] \times D_{R}}|\gamma(t, x)| .
\end{aligned}
$$

Lemma 6.5.2. We can take $0<T<T_{1}$ and $0<R<R_{1}$ so that the following conditions are satisfied:

$$
\begin{aligned}
& A_{1} r_{1}+A_{2} r_{2} \leq \frac{a}{2}, \\
& B \varphi(T)+\frac{B_{1} r_{1}+B_{2} r_{2}}{a}+\frac{B_{2} L r_{1}}{a^{2}}<\frac{R}{4}, \\
& C \leq \frac{a}{2} .
\end{aligned}
$$

Proof. By (6.5.2) we have

$$
\begin{aligned}
& \lim _{T \rightarrow 0} r_{1}=o\left(R^{2}\right) \quad(\text { as } R \longrightarrow+0), \\
& \lim _{T \rightarrow 0} r_{2}=o(R) \quad(\text { as } R \longrightarrow+0)
\end{aligned}
$$

and so by taking $T>0$ and $R>0$ sufficiently small we have the conditions (6.5.5) and (6.5.6). Since $\left|b_{1}(t, x)\right| \leq B_{1} r_{1}+B_{2} r_{2}$ on $(0, T] \times D_{R}$ we have

$$
\begin{aligned}
& \lim _{T \rightarrow 0} \sup _{(0, T] \times D_{R}}\left|b_{1}(t, x)\right|=o(R) \quad(\text { as } R \longrightarrow+0), \\
& \lim _{T \rightarrow 0} \sup _{(0, T] \times D_{R}}\left|\left(\partial b_{1} / \partial x\right)(t, x)\right|=o(1) \quad(\text { as } R \longrightarrow+0) .
\end{aligned}
$$


Therefore, by taking $T>0$ and $R>0$ sufficiently small we have the condition $\left|\left(\partial b_{1} / \partial x\right)(t, x)\right| \leq a / 4$ on $(0, T] \times D_{R}$. Since $|b(t, x)| \leq B \mu(t)$ holds on $\left.\left[0, T_{0}\right] \times D_{R_{0}}\right)$, by taking $T>0$ sufficiently small we have $|(\partial b / \partial x)(t, x)| \leq$ $a / 4$ on $(0, T] \times D_{R}$. Since $c(t, x)=\left(\partial b_{1} / \partial x\right)(t, x)+(\partial b / \partial x)(t, x)$ we have the condition (6.5.7).

Corollary 6.5.3. Under the situation in Lemma 6.5.2 we have

$$
\begin{aligned}
& \operatorname{Re}\left(\lambda(t, x)+\lambda_{1}(t, x)\right) \geq a \quad \text { on }(0, T] \times D_{R}, \\
& \operatorname{Re}\left(\lambda(t, x)+\lambda_{1}(t, x)+c(t, x)\right) \geq a \quad \text { on }(0, T] \times D_{R} .
\end{aligned}
$$

Proof. Since $\left|\lambda_{1}(t, x)\right| \leq A_{1} r_{1}+A_{2} r_{2}$ and $|c(t, x)| \leq C$ hold on $(0, T] \times D_{R}$, by (6.5.5) and (6.5.7) we have the result.

Step 3: From now, we take $T>0$ and $R>0$ as in Lemma 6.5.2, and fix them. For $\xi \in D_{R}$, let us consider the initial value problem

$$
\frac{d x}{d t}=-\frac{b(t, x)+b_{1}(t, x)}{t},\left.\quad x\right|_{t=T}=\xi .
$$

Let $\left(t_{\xi}, T\right]$ be the maximal existence domain of the solution $x(t)$ of $(6.5 .8)$. Set

$$
\begin{array}{ll}
u^{*}(t)=u(t, x(t)), & q^{*}(t)=q(t, x(t)), \\
\lambda^{*}(t)=\lambda(t, x(t)), & \lambda_{1}^{*}(t)=\lambda_{1}(t, x(t)), \\
c^{*}(t)=c(t, x(t)), & \gamma^{*}(t)=\gamma(t, x(t)):
\end{array}
$$

these functions are well-defined on $\left(t_{\xi}, T\right]$. By (6.5.3) and (6.5.4) we have

$$
\begin{aligned}
& t \frac{d u^{*}}{d t}=\left(\lambda^{*}(t)+\lambda_{1}^{*}(t)\right) u^{*} \quad \text { on }\left(t_{\xi}, T\right], \\
& t \frac{d q^{*}}{d t}=\left(\lambda^{*}(t)+\lambda_{1}^{*}(t)+c^{*}(t)\right) q^{*}+\gamma^{*}(t) u^{*} \quad \text { on }\left(t_{\xi}, T\right] .
\end{aligned}
$$

Lemma 6.5.4. In the above situation, for any $t \in\left(t_{\xi}, T\right]$ we have

$$
\begin{aligned}
& \left|u^{*}(t)\right| \leq r_{1}\left(\frac{t}{T}\right)^{a}, \\
& \left|q^{*}(t)\right| \leq r_{2}\left(\frac{t}{T}\right)^{a}+L r_{1}\left(\frac{t}{T}\right)^{a} \log \left(\frac{T}{t}\right), \\
& |x(t)| \leq|\xi|+B \varphi(T)+\frac{B_{1} r_{1}+B_{2} r_{2}}{a}+\frac{B_{2} L r_{1}}{a^{2}} .
\end{aligned}
$$

Proof. We set

$$
E(t)=\exp \left[\int_{t}^{T} \frac{\lambda^{*}(s)+\lambda_{1}^{*}(s)}{s} d s\right], \quad t_{\xi}<t \leq T .
$$


Then, by (6.5.9) we have

$$
\frac{d}{d t}\left(u^{*}(t) E(t)\right)=0, \quad t_{\xi}<t \leq T,
$$

and so by integrating this from $t$ to $T$ we have $u^{*}(T)-u^{*}(t) E(t)=0$, that is,

$$
u^{*}(t)=u^{*}(T) \exp \left[-\int_{t}^{T} \frac{\lambda^{*}(s)+\lambda_{1}^{*}(s)}{s} d s\right] \quad \text { on }\left(t_{\xi}, T\right] .
$$

Since $\left|u^{*}(T)\right| \leq r_{1}$ and $\operatorname{Re}\left(\lambda^{*}(t)+\lambda_{1}^{*}(t)\right) \geq a$ on $\left(t_{\xi}, T\right]$, we have

$$
\left|u^{*}(t)\right| \leq r_{1} \exp \left[-\int_{t}^{T} \frac{a}{s} d s\right]=r_{1}\left(\frac{t}{T}\right)^{a} \quad \text { on }\left(t_{\xi}, T\right] .
$$

This proves (6.5.11). Similarly, if we set

$$
E_{1}(t)=\exp \left[\int_{t}^{T} \frac{\lambda^{*}(s)+\lambda_{1}^{*}(s)+c^{*}(s)}{s} d s\right], \quad t_{\xi}<t \leq T,
$$

by the same argument we have

$$
\begin{aligned}
q^{*}(t)= & q^{*}(T) \exp \left[-\int_{t}^{T} \frac{\lambda^{*}(s)+\lambda_{1}^{*}(s)+c^{*}(s)}{s} d s\right] \\
& -\int_{t}^{T} \exp \left[-\int_{t}^{\tau} \frac{\lambda^{*}(s)+\lambda_{1}^{*}(s)+c^{*}(s)}{s} d s\right] \frac{\gamma^{*}(\tau) u^{*}(\tau)}{\tau} d \tau .
\end{aligned}
$$

Since $\left|q^{*}(T)\right| \leq r_{2}, \operatorname{Re}\left(\lambda^{*}(t)+\lambda_{1}^{*}(t)+c^{*}(t)\right) \geq a$ and $\left|\gamma^{*}(t)\right| \leq L$ on $\left(t_{\xi}, T\right]$, by (6.5.11) we have

$$
\begin{aligned}
\left|q^{*}(t)\right| & \leq r_{2}\left(\frac{t}{T}\right)^{a}+\int_{t}^{T}\left(\frac{t}{\tau}\right)^{a} \frac{L r_{1}(\tau / T)^{a}}{\tau} d \tau \\
& =r_{2}\left(\frac{t}{T}\right)^{a}+L r_{1}\left(\frac{t}{T}\right)^{a} \log \left(\frac{T}{t}\right) \quad \text { on }\left(t_{\xi}, T\right] .
\end{aligned}
$$

This proves (6.5.12).

Let us show (6.5.13). Since $x(t)$ is a solution of (6.5.8), we have

$$
x(t)=\xi+\int_{t}^{T} \frac{b(\tau, x(\tau))+b_{1}(\tau, x(\tau))}{\tau} d \tau .
$$

Since $\left|b_{1}(t, x)\right| \leq B_{1}|u(t, x)|+B_{2}|q(t, x)|$ is known on $\left(0, T_{1}\right] \times D_{R_{1}}$, by (6.1.3), (6.5.11) and (6.5.12) we have

$$
|b(t, x(t))|+\left|b_{1}(t, x(t))\right| \leq \Phi(t) \quad \text { on }\left(t_{\xi}, T\right]
$$

with

$$
\Phi(t)=B \mu(t)+B_{1} r_{1}\left(\frac{t}{T}\right)^{a}+B_{2} r_{2}\left(\frac{t}{T}\right)^{a}+B_{2} L r_{1}\left(\frac{t}{T}\right)^{a} \log \left(\frac{T}{t}\right) .
$$


Hence, we obtain

$$
\begin{aligned}
|x(t)| & \leq|\xi|+\int_{t}^{T} \frac{\Phi(\tau)}{\tau} d \tau \\
& \leq|\xi|+B \varphi(T)+\frac{B_{1} r_{1}+B_{2} r_{2}}{a}+\frac{B_{2} L r_{1}}{a^{2}} \quad \text { on }\left(t_{\xi}, T\right] .
\end{aligned}
$$

This proves (6.5.13).

Corollary 6.5.5. If $|\xi|<3 R / 4$, we have $t_{\xi}=0$.

Proof. If $|\xi|<3 R / 4$, by (6.5.6) and (6.5.13) we have

$$
|x(t)| \leq \frac{3 R}{4}+B \varphi(T)+\frac{B_{1} r_{1}+B_{2} r_{2}}{a}+\frac{B_{2} L r_{1}}{a^{2}}<R
$$

on $\left(t_{\xi}, T\right]$. If $t_{\xi}>0$, this estimate asserts that the solution $x(t)$ can be extended to $\left(t_{\xi}-\delta, T\right]$ for some $\delta>0$. But, this contradicts the condition that $\left(t_{\xi}, T\right]$ is a maximal existence domain. Hence, we have $t_{\xi}=0$.

Step 4: We denote by $\phi_{1}(t ; T, \xi)$ the unique solution of (6.5.8): we have

$$
\phi_{1}(t ; T, \xi)=\xi+\int_{t}^{T} \frac{b\left(\tau, \phi_{1}(\tau ; T, \xi)\right)+b_{1}\left(\tau, \phi_{1}(\tau ; T, \xi)\right)}{\tau} d \tau .
$$

Set

$$
\mathcal{D}=\bigcup_{\xi \in D_{3 R / 4}}\left\{\left(t, \phi_{1}(t ; T, \xi)\right) ; 0<t \leq T\right\} .
$$

Then, by Lemma 6.5.4 we have

$$
\begin{aligned}
& |u(t, x)| \leq r_{1}\left(\frac{t}{T}\right)^{a} \quad \text { on } \mathcal{D}, \\
& |q(t, x)| \leq r_{2}\left(\frac{t}{T}\right)^{a}+L r_{1}\left(\frac{t}{T}\right)^{a} \log \left(\frac{T}{t}\right) \quad \text { on } \mathcal{D} .
\end{aligned}
$$

Therefore, to get the condition $u(t, x) \in X_{1}^{+}$it is enough to prove the following result.

Lemma 6.5.6. We have the condition

$$
(0, T] \times D_{R / 4} \subset \mathcal{D} .
$$

Proof. To show this, it is sufficient to porve the following assertion: for any $\left(t_{0}, x_{0}\right) \in(0, T) \times D_{R / 4}$ there is a unique $\xi \in D_{R / 2}$ such that $\phi_{1}\left(t_{0} ; T, \xi\right)=x_{0}$ holds, that is,

$$
x_{0}=\xi+\int_{t_{0}}^{T} \frac{b\left(\tau, \phi_{1}(\tau ; T, \xi)\right)+b_{1}\left(\tau, \phi_{1}(\tau ; T, \xi)\right)}{\tau} d \tau
$$


holds. For simplicity, we set

$$
G(t, \xi)=b\left(t, \phi_{1}(t ; T, \xi)\right)+b_{1}\left(t, \phi_{1}(t ; T, \xi)\right) \in X_{0}\left((0, T] \times D_{3 R / 4}\right) .
$$

By (6.5.14) and the Cauchy's inequality, we have

$$
\begin{aligned}
& |G(t, \xi)| \leq \Phi(t) \quad \text { on }(0, T] \times D_{3 R / 4}, \\
& \left|\frac{\partial G}{\partial \xi}(t, \xi)\right| \leq \frac{\Phi(t)}{R / 4} \quad \text { on }(0, T] \times D_{R / 2} .
\end{aligned}
$$

Therefore, we have the Lipschitz condition:

$$
\left|G\left(t, \xi_{1}\right)-G\left(t, \xi_{2}\right)\right| \leq \frac{\Phi(t)}{R / 4}\left|\xi_{1}-\xi_{2}\right|
$$

for any $\left(t, \xi_{i}\right) \in(0, T] \times D_{R / 2}(i=1,2)$. We set also

$$
K=B \varphi(T)+\frac{B_{1} r_{1}+B_{2} r_{2}}{a}+\frac{B_{2} L r_{1}}{a^{2}}:
$$

by (6.5.6) we have $K<R / 4$ and under the notation (6.5.15) we have

$$
\int_{0}^{T} \frac{\Phi(\tau)}{\tau} d \tau=K
$$

Let us solve (6.5.16), that is,

$$
\xi=x_{0}-\int_{t_{0}}^{T} \frac{G(\tau, \xi)}{\tau} d \tau .
$$

To solve this equation, we use the method of successive approximations: the approximate solutions $\xi_{n}(n=0,1,2, \ldots)$ are defined by $\xi_{0}=x_{0}$ and

$$
\xi_{n}=x_{0}-\int_{t_{0}}^{T} \frac{G\left(\tau, \xi_{n-1}\right)}{\tau} d \tau, \quad n \geq 1 .
$$

Since $\xi_{0}=x_{0} \in D_{R / 4}$, we see that $G\left(\tau, \xi_{0}\right)$ is well-defined. Then, $\xi_{1}$ is well-defined and

$$
\left|\xi_{1}\right| \leq\left|x_{0}\right|+\int_{t_{0}}^{T} \frac{\Phi(\tau)}{\tau} d \tau<R / 4+K<R / 4+R / 4=R / 2 .
$$

In addition, we have

$$
\left|\xi_{1}-\xi_{0}\right| \leq\left|\int_{t_{0}}^{T} \frac{G\left(\tau, \xi_{0}\right)}{\tau} d \tau\right| \leq \int_{t_{0}}^{T} \frac{\Phi(\tau)}{\tau} d \tau=K .
$$

In this way, by a standard argument we can see that $\xi_{n} \in D_{R / 4+K} \subset D_{R / 2}$ $(n=0,1,2, \ldots)$ are well-defined and we have

$$
\left|\xi_{n}-\xi_{n-1}\right| \leq K\left(\frac{K}{R / 4}\right)^{n-1}, \quad n=1,2, \ldots
$$


Since $K /(R / 4)<1$ is supposed, we see that $\xi_{n}$ is convergent (as $n \longrightarrow \infty$ ) to a solution $\xi \in D_{R / 2}$ of (6.5.17). This shows the existence of a solution $\xi \in D_{R / 2}$ of (6.5.16).

The uniqueness of the solution can be proved in the same way.

This completes the proof of Theorem 6.5.1,

\section{References}

[1] Ch. Briot, J. CL. Bouquet, Recherches sur les propriétés des fonctions définies par des équations différentielles, J. Ecole Polytech. 21 (1856), 133-197.

[2] R. GÉrARD, Etude locale des équations différentielles de la forme $x y^{\prime}=$ $f(x, y)$ au voisinage de $x=0$, J. Fac. Sci. Univ. Tokyo Sect. IA, Math. 36 (1989), 729-752.

[3] R. GÉrard, H. Tahara, Holomorphic and singular solutions of nonlinear singular first order partial differential equations, Publ. Res. Inst. Math. Sci. 26 (1990), no. 6, 979-1000.

[4] R. GÉrard, H. TAhara, Singular nonlinear partial differential equations, Aspects of Mathematics, E28, Friedr. Vieweg \& Sohn, Braunschweig, 1996.

[5] E. Hille, Ordinary differential equations in the complex domain, John Wiley and Sons, 1976.

[6] M. Hukuhara, T. Kimura, T. Matuda, Équations différentielles ordinaires du premier ordre dans le champ complexe, Publications of the Mathematical Society of Japan, 7, The Mathematical Society of Japan, Tokyo, 1961.

[7] K. Iwasaki, H. Kimura, S. Shimomura, M. Yoshida, From Gauss to Painlevé, A Modern Theory of Special Functions, Aspects of Mathematics, E16, Friedr. Vieweg \& Sohn, Braunschweig, 1991.

[8] F. Li, On systems of partial differential equations of Briot-Bouquet type, Bull. Aust. Math. Soc. 98 (2018), 122-133.

[9] J. E. C. Lope, M. P. Roque, H. Tahara, On the unique solvability of certain nonlinear singular partial differential equations, Z. Anal. Anwend. 31 (2012), no. 3, 291-305.

[10] M. Nagumo, Uber das Anfangswertproblem partieller Differentialgleichungen, Japan. J. Math. 18 (1941), 41-47. 
[11] L. Nirenberg, An abstract form of the nonlinear Cauchy-Kowalewski theorem, J. Differential Geometry 6 (1972), 561-576.

[12] G. Sansone, R. Conti, Non-linear differential equations. Revised edition, International Series of Monographs in Pure and Applied Mathematics, Vol. 67, A Pergamon Press Book. The Macmillan Co. New York, 1964.

[13] H. TAhara, Uniqueness of the solution of nonlinear singular first order partial differential equations, to appear in J. Math. Soc. Japan Advanced publication: https://projecteuclid.org/euclid.jmsj/1589184011

[14] F. Treves, An abstract nonlinear Cauchy-Kovalevska theorem, Trans. Amer. Math. Soc. 150 (1970), 77-72.

[15] A. Wintner, Asymptotic integration constants in the singularity of Briot-Bouquet, Amer. J. Math. 68 (1946), 293-300.

[16] H. Yamazawa, Singular solutions of the Briot-Bouquet type partial differential equations, J. Math. Soc. Japan 55 (2003), no. 3, 617-632.

[17] H. Yamazawa, Holomorphic and singular solutions of $q$-differencedifferential equations of Briot-Bouquet type, Funkcialaj Ekvacioj, 59 (2016), no. 2, 185-197. 